Actualidades en Psicología, 32(125), 2018, 113-131

ISSN 2215-3535

http://revistas.ucr.ac.cr/index.php/actualidades

DOI: https://doi.org/10.15517/ap.v32i125.31976

\title{
Estrategias argumentativas en situaciones naturales de interacción en el aula de nivel preescolar
}

\author{
Argumentative Strategies During Interaction in \\ Natural Settings in Preschool Classrooms
}

\author{
Genis Jiménez Ramírez ${ }^{1}$ \\ Lizbeth Vega Pérez ${ }^{2}$ \\ Universidad Nacional Autónoma de México, México
}

\begin{abstract}
Resumen. El objetivo de este estudio fue analizar las estrategias argumentativas empleadas por niños preescolares en situaciones naturales de interacción en el aula. Participaron 60 niños entre los 3 y 5 años de una Estancia Infantil de la Ciudad de México. La investigación fue cualitativa y se realizó un proceso de observación sistemática no participante en tres momentos del día: asamblea, actividad pedagógica y juego libre con juguetes. Los resultados mostraron que en las conversaciones que tenían con sus maestras y entre pares, los niños generaron 10 estrategias argumentativas: referente de autoridad, descripción, comparación, analogía, generalización, narración, anticipación, causalidad, oposición y propuesta alternativa. Estos resultados aportan evidencia empírica en torno a la argumentación en la primera infancia.
\end{abstract}

Palabras Clave. Estrategias argumentativas, argumentación, situaciones naturales, interacción social, niños preescolares.

Abstract. The goal of this study was to analyze the argumentative strategies used by preschool children in natural situations of interaction in the classroom. Participants 60 children between 3 and 5 years old from a Day Care Center in Mexico City. The research was qualitative and a non-participant systematic observation process was conducted at three moments of the day: the assembly, the pedagogical activity and the free play with toys. The results showed that during conversation with their teachers and among peers, children generated 10 argumentative strategies: reference of authority, description, comparison, analogy, generalization, narration, anticipation, causality, opposition and alternative proposal. These results provide empirical evidence about argumentation in early childhood.

Keywords. Argumentative strategies, argumentation, natural settings, social interaction, preschool children.

${ }^{1}$ Genis Yaisuri Jiménez Ramírez. Universidad Nacional Autónoma de México, México. Dirección Postal: Av. Universidad 3094, Col. Copilco, CP. 04510. Del. Coyoacán, Cuidad de México, México. E-mail: genisjramirez@gmail.com

${ }^{2}$ Lizbeth Obdulia Vega Pérez. Universidad Nacional Autónoma de México, México. E-mail: lvega@unam.mx

\section{@ $\odot \Theta \Theta$}

Esta obra está bajo una licencia de Creative Commons Reconocimiento-NoComercial-SinObraDerivada 4.0 Internacional. 


\section{Introducción}

La argumentación es una actividad discursiva fundamental en el desarrollo social, lingüístico y cognoscitivo de los seres humanos (Kuhn, Hemberger, \& Khait, 2016; Zadunaisky \& Blum-Kulka, 2010). Desde la perspectiva psicológica en la cual se enmarca este estudio, son importantes las contribuciones de autores como Kuhn et al. (2016), Mercer y Littleton (2007), Mercer (2011) y Muller, Perret-Clermont, Tartas y Iannaccone (2009). Todos ellos han conceptualizado este constructo como una práctica que se sitúa en el contexto institucional, histórico y cultural en el que ocurren las interacciones cotidianas de las personas y que responde a la necesidad de comunicación, verificación y posicionamiento frente a los otros.

Al respecto, Kuhn (1993) definió la argumentación como "una actividad social en la cual dos o más personas defienden y comparan argumentos que apoyan o se oponen a diversas posiciones" (p. 5). Asimismo, Kuhn y Udell (2003) señalaron que el argumento es un componente central en la actividad argumentativa, y lo definieron como una cadena interna de razonamiento que comprende, específicamente, el punto de vista y su justificación.

La evidencia empírica vinculada con el estudio de la argumentación en la primera infancia presenta resultados contradictorios (Cisterna \& Garayzábal, 2016). En torno a esto, existen líneas de trabajo que definen la argumentación como un proceso complejo vinculado con la lógica, la construcción de inferencias y demostraciones formalmente válidas que solo se manifiesta en la adolescencia y la adultez (Coirier \& Golder, 1993; Dolz \& Pasquier, 2000; Silvestri, 2001).

Contrario a lo anterior, se han llevado a cabo distintas investigaciones, fundamentalmente desde la perspectiva lingüística, la cual analiza la estructura lógica de un argumento, así como las evidencias empleadas por los niños para justificar un punto de vista. Estos estudios coinciden en afirmar que entre los 3 y 4 años de edad es posible identificar la emergencia de la actividad argumentativa en la infancia, específicamente en situaciones de conflicto en el hogar o la escuela (Banks-Leite, 1997; Eisenberg, 1987; Köymen, Rosenbaum, \& Tomasello, 2014; Kuo, 2011; Macedo, 2011; Migdalek \& Rosemberg, 2013; Migdalek, Santíbañez, \& Rosemberg, 2014a; Stein \& Albro, 2001; Zadunaisky \& Blum-Kulka, 2010).

Cabe mencionar que una línea de investigación importante en el estudio de la argumentación infantil son las estrategias argumentativas, las cuales han sido definidas como el soporte verbal que emplean las personas para hacer más contundente un discurso (Kuhn \& Udell, 2003). La importancia de estudiar las estrategias argumentativas en la primera infancia se vincula con tres aspectos esenciales.

Primero, se ha documentado el vínculo entre la construcción de una estrategia y la forma de razonamiento que tiene lugar en la mente de las personas. Por lo tanto, cuando un niño justifica un punto de vista emplea habilidades cognoscitivas como descripción, comparación, relación, generalización y síntesis, las cuales son indicadoras del desarrollo cognoscitivo en esta etapa (Muller et al., 2009; Kuhn \& Udell, 2003).

En segundo lugar, la construcción de una estrategia implica la habilidad de descentramiento; es decir, la capacidad de considerar el punto de vista del interlocutor y reconocer que 
este tiene ideas y conocimientos sobre el tema de la conversación que también pueden ser aceptables. En consecuencia, los niños se ven en la necesidad de argumentar para defender su punto de vista (Muller et al., 2009).

Por último, la evidencia empírica ha constatado que la construcción de estrategias argumentativas en la primera infancia no se explica solo por la edad de los niños, sino que están fuertemente relacionadas con las situaciones de interacción en el hogar y la escuela, que crean espacios de diálogo argumentativo. Por consiguiente, es importante que los maestros que laboran en el nivel preescolar conozcan los tipos de estrategias argumentativas que pueden construir sus alumnos en esta etapa y promuevan su emergencia, mediante el diseño de planeaciones y situaciones de interacción específicas (Rapanta, García-Mila, \& Gilabert, 2013).

Dentro de los estudios antecedentes, se encuentran los aportes de Eisenberg (1987) quien realizó un seguimiento a dos niñas desde los 2 a los 3 años para observar los diferentes tipos de estrategias persuasivas empleadas en las interacciones que tenían con sus pares, pa padres y otros adultos. Encontró, en sus resultados, que las participantes utilizaron las siguientes estrategias para sostener sus puntos de vista: insistencia, repetición y la amenaza.

Kuo (2011) estudió la utilización de estrategias argumentativas en niños entre los 3 y 4 años. Los resultados indicaron que los participantes empleaban diversas estrategias argumentativas para oponerse o buscar convencer a otros; asimismo, identificó que la fuente de conflictos más común era por la posesión de un objeto (juguetes). Las estrategias argumentativas más utilizadas tanto por niños como niñas fueron: la insistencia, la repetición y el apoyo verbal.

En el contexto de jardines infantiles, González (2007) analizó los procesos de argumentación conjunta entre maestras y alumnos de nivel preescolar a partir de la lectura de tres cuentos infantiles y encontró que los niños emplearon las estrategias de acuerdo, desacuerdo o confrontación; también, que utilizaban justificaciones para sustentar su punto de vista. Asimismo, encontró que el diálogo argumentativo en los niños era más frecuente cuando los maestros les formulaban preguntas y les solicitaban explicaciones respecto a lo sucedido en los cuentos, por lo que resaltó la importancia del rol del maestro como generador de situaciones argumentativas dentro del aula.

Dovingo (2016) realizó un estudio para analizar la emergencia de la argumentación en las interacciones maestro-alumno y entre pares en el nivel preescolar. En sus resultados, identificó que las interacciones maestro-alumno se basaban en una relación más formal que es intrínsecamente asimétrica, siendo el maestro quien generalmente proponía el tema de conversación, lideraba el diálogo y formulaba las preguntas con la finalidad de que los alumnos dieran sus puntos de vista y construyeran argumentos. En cambio, también encontró que la interacción entre pares era simétrica y se presentaban en ella intercambios comunicativos típicamente informales que emergían en las situaciones de juego.

En Latinoamérica sobresalen los estudios de Migdalek y Rosemberg (2013) y Migdalek et al. (2014a; 2014b), quienes crearon y validaron un sistema de estrategias argumentativas infantiles en situaciones de disputa en el contexto del hogar y la escuela. Clasificaron dichas estrategias de acuerdo con el principio de complejidad, siendo la reiteración la única 
estrategia simple. Del mismo modo, identificaron las siguientes estrategias complejas: narración, anticipación, generalización, descripción, apelación a la autoridad, cortesía y propuesta alternativa.

En México, los trabajos sobre las estrategias argumentativas en niños preescolares son escasos. En la revisión realizada solo se reportó un estudio realizado por Márquez (2016). En su investigación, encontró que los niños entre los 3 y 4 años, emplearon las estrategias argumentativas de: reiteración, descripción, anticipación y propuesta alternativa en situaciones en las que debían solucionar un conflicto.

En síntesis, un aspecto en común de los estudios empíricos sobre las estrategias argumentativas es que se ha focalizado en investigar este constructo en situaciones de disputa que se dan en el nivel preescolar o mediante actividades diseñadas para que los niños argumenten como la lectura de cuentos. Además, estas investigaciones se han llevado a cabo, fundamentalmente, desde una perspectiva lingüística que se focaliza en el análisis de la estructura de la estrategia argumentativa; es decir, punto de vista y justificación.

Sin embargo, no se ahonda en otros aspectos igualmente importantes como la diversidad de situaciones de interacción en la que emergió la estrategia, el proceso de razonamiento seguido por el niño para construir una estrategia, así como otras intenciones argumentativas que pudiera tener cuando participa en un diálogo argumentativo, más allá de querer persuadir o convencer. De ahí que el objetivo esta investigación fue identificar las estrategias argumentativas de un grupo de niñas y niños mexicanos de nivel preescolar, en situaciones naturales de interacción en el aula de clase.

\section{Método}

\section{Participantes}

Participaron en este estudio 60 niños de nivel preescolar con edades entre los 3 años y los 5 años 11 meses, quienes se encontraban inscritos en una Estancia Infantil del Instituto de Seguridad y Servicios Sociales de los Trabajadores del Estado (ISSSTE) ubicada en la zona sur de la Ciudad de México. Esta cuenta con equipo interdisciplinario compuesto por profesionales con experiencia en la atención de la primera infancia. Posee, también, material didáctico, juguetes, juegos de construcción y espacios lúdicos dentro del aula de clase que contribuyen a potenciar en los alumnos su desarrollo integral.

Específicamente, la muestra de niños se distribuyó en 3 grupos de nivel preescolar: grupo de primero (22 alumnos: 9 niñas y 13 niños, $M=3$ años 5 meses); grupo de segundo (22 alumnos: 17 niñas y 5 niños, $M=4$ años 3 meses); y el grupo de tercero (16 alumnos: 10 niñas y 6 niños, $M=5$ años 6 meses). También participaron 6 maestras (3 maestras titulares y 3 maestras de apoyo).

Cabe mencionar que en cada grado escolar había dos maestras: una maestra titular y una de apoyo. La edad promedio de las maestras fue de 37 años y, en relación con su tiempo de experiencia docente en el nivel preescolar, se obtuvo un promedio de 12 años.

\section{Unidad de análisis}


La unidad de análisis fue el evento argumentativo, el cual fue definido por las autoras de este estudio como un momento de la conversación en el aula de clase en el que surgía un punto de vista y su respectiva justificación. Cabe mencionar que es precisamente en el tipo de justificación que aportaron los niños que fue posible identificar las estrategias argumentativas empleadas como sustento y evidencia de sus afirmaciones.

\section{Tipo y diseño de investigación}

El tipo de investigación fue cualitativa y específicamente se realizó un análisis cualitativo del contenido de las conversaciones que surgieron en las situaciones naturales de interacción maestro-alumno y entre pares en el aula de clase (Álvarez-Gayou, 2011; Coffey \& Atkinson, 2004; Flick, 2007). El diseño de esta investigación se construyó después de un proceso de familiarización con las actividades cotidianas de la Estancia Infantil, el cual sirvió para seleccionar tres grupos de nivel preescolar: primero, segundo y tercero.

De manera específica, en cada uno de los grupos se realizó un proceso de observación sistemática no participante (Ruiz, 1996), que se estructuró a partir de sesiones de observación planeadas con una regularidad y secuencia establecidas; lo anterior con el objetivo de registrar las situaciones naturales de interacción que se documentaron en este estudio. Las investigadoras asumieron el rol de observador completo (Ruiz, 1996) que consistió en abstenerse de participar o emitir algún comentario en las interacciones que estaban teniendo los participantes en el aula de clase.

\section{Materiales e Instrumentos}

Se empleó una bitácora de observación de los eventos argumentativos en la que se registró: (1) el tipo de interacción: maestro-alumno o entre pares; (2) el inicio de evento argumentativo: turno conversacional específico de la conversación que antecede a que un niño presente una estrategia argumentativa; (3) la intención argumentativa: propósito que persigue el niño cuando emplea una determinada estrategia; (4) las estrategias argumentativas: tipo de justificaciones verbales que emplea un niño como evidencia de su punto de vista; y (5) el cierre del evento argumentativo: turno conversacional por medio del cual se concluye el evento argumentativo. La bitácora se complementó con el registro de grabación de las situaciones naturales de interacción en el aula de clase y las notas de campo.

\section{Procedimiento}

Para llevar a cabo este estudio, se contó con el consentimiento informado del Departamento de Acción Social, Cultural y Deportiva del ISSSTE, responsable del funcionamiento de las Estancias Infantiles en la Ciudad de México. Asimismo, esta investigación se realizó siguiendo los lineamientos del Código Ético del Psicólogo (Sociedad Mexicana de Psicología, 2009) y los Principios éticos en el trabajo con niños (National Association for the Education of Young Children, 2011).

El proceso de observación sistemática no participante tuvo una duración de diez meses durante el ciclo escolar 2016-2017 en la que se realizaron 150 horas de grabación en tres tipos de actividades, como se detalla a continuación.

La asamblea. Todos los niños del grupo se distribuían en círculo con su maestra; generalmente, 
ella formulaba una pregunta o tema de discusión y los niños comenzaban a participar aportando sus puntos de vista. En promedio, la asamblea tenía una duración de 15 minutos.

La actividad pedagógica. Los alumnos se ubicaban en mesas integradas por cinco niños -cuatro mesas en total por grupo- en la que se observaba la interacción de cada mesa por un lapso de cinco minutos. El tiempo total de duración de esta actividad era de 30 minutos.

El juego libre con juguetes. Los niños se distribuían de manera aleatoria en el espacio del aula de clase y allí de manera espontánea elegían juguetes disponibles en la sala y formaban parejas o grupos de 3 a 4 niños. Se observaba el juego libre por un tiempo de 30 minutos.

\section{Análisis de datos}

Se realizó un análisis cualitativo del contenido a través de un proceso de codificación mixta (Rodríguez, Lorenzo, \& Herrera, 2005), el cual consistió en una revisión de estudios antecedentes sobre las estrategias argumentativas verbales en niños preescolares, en los que se identificaron las siguientes estrategias: referente de autoridad, descripción, narración, generalización, anticipación y propuesta alternativa (Migdalek \& Rosemberg, 2013; Migdalek et al., 2014a; 2014b; Márquez, 2016); las estrategias de analogía y comparación (Martí, 1978) y la estrategia de oposición (González, 2014; Kuo, 2011).

Posteriormente, se analizaron los eventos argumentativos obtenidos en este estudio con base en las estrategias identificadas en el estado del arte, las cuales fueron codificadas con el apoyo del Programa de análisis de datos cualitativos ATLAS.ti8 y siguiendo las reglas de categorización propuesta por Kerlinger y Lee (2002): (1) categorías definidas de acuerdo con el objetivo de la investigación, (2) principio de exhaustividad, (3) principio de exclusión e independencia, y (4) principio de clasificación.

\section{Control de calidad de datos}

Para alcanzar los criterios de credibilidad y validez de los datos, se llevaron a cabo varios procedimientos. Primero, la triangulación metodológica (bitácora de registro de eventos argumentativos, grabación y notas de campo); en segundo lugar, la triangulación teórica (codificación mixta a partir de la revisión del estado del arte sobre estrategias argumentativas en primera infancia). Finalmente, se realizó el proceso de confirmabilidad (Ruiz, 1996), en el cual participaron cuatro jueces expertos en el estudio de la argumentación en primera infancia y dos jueces expertos en la metodología cualitativa.

\section{Resultados}

El análisis cualitativo del contenido con el apoyo del programa ATLAS.ti8, evidenció que los niños de nivel preescolar que participaron en la investigación generaron diversas estrategias argumentativas en las situaciones naturales de interacción documentadas en este estudio. Estas estrategias fueron validadas a partir de un proceso de confirmabilidad por jueces expertos, que consistió en presentar a los jueces 20 eventos argumentativos en los que surgieron estrategias argumentativas y ellos emitieron un juicio de acuerdo o desacuerdo con el proceso de codificación de cada estrategia, según los siguientes criterios: (1) grado de relevancia, suficiencia y pertinencia de cada estrategia, y (2) el cumplimiento de las reglas de categorización propuestas por Kerlinger y Lee (2002). Los jueces también 
identificaron turnos conversacionales dentro de los eventos argumentativos que no estaban categorizados y a los que se les debían incluir algún tipo de estrategia.

Las observaciones y valoraciones cualitativas de los seis jueces fueron incorporadas al proceso de categorización de este estudio. El resultado final de este proceso de confirmabilidad fue la obtención de diez categorías de estrategias argumentativas verbales empleadas por lo niños participantes para justificar sus puntos de vista: referente de autoridad, descripción, comparación, analogía, generalización, narración, anticipación, causalidad, oposición y propuesta alternativa. La tabla 1 muestra la descripción de cada una de las estrategias, su definición y los ejemplos que fueron extraídos de los eventos argumentativos documentados en este estudio.

Un análisis más fino de cada una de las estrategias permitió generar un Sistema de estrategias argumentativas que presenta una relación dinámica con los siguientes elementos: situaciones naturales de interacción en el aula de clase, inicio del evento argumentativo, intención argumentativa y cierre del evento (véase figura 1).

Situaciones naturales de interacción en el aula

En un nivel más general del sistema, se encuentran las situaciones naturales de interacción en el aula de clase (maestro-alumno y entre pares) que permiten delimitar el contexto de las conversaciones de los participantes en el que emergen las estrategias argumentativas. En torno a la interacción maestro-alumno, se obtuvo que las maestras participantes posibilitaban las argumentaciones de los niños dando apertura a las conversaciones a través de preguntas específicas sobre temas curriculares o situaciones hipotéticas, por ejemplo “QQué pasaría si todos fuéramos iguales?”.

También, se identificaron otro tipo de intervenciones vinculadas con la actividad argumentativa en el aula de clase. Entre ellas, retomar lo dicho por los niños para dar continuidad al tema de conversación, y realizar comentarios, aclaraciones y correcciones en relación con la conversación que estaban teniendo con sus alumnos.

Por su parte, la interacción entre pares, en la que también emergieron estrategias argumentativas, se llevó a cabo fundamentalmente en las situaciones de juego simbólico en la que los niños representaban diferentes roles como maestros, doctores o padres de familia. Cabe mencionar que dichos juegos guardaban relación con los juguetes que tenían disponibles los niños en el aula de clase: muñecos para representar los roles de mamá e hijo, estetoscopios e inyecciones para escenificar los roles de doctor y paciente. Asimismo, en la interacción entre pares la emocionalidad y el lenguaje no verbal (particularmente cambios en el tono de voz y gestos) se hicieron más notorios en las conversaciones, lo que les permitía a los niños enfatizar sus puntos de vista.

\section{Inicio del evento argumentativo}

El siguiente elemento del sistema corresponde con el turno conversacional que antecede a que un niño presente una estrategia argumentativa. En las interacciones maestro-alumno que se documentaron, generalmente fue la maestra quien tomó el rol de moderadora de la conversación, asignando turnos para hablar a los niños y generando preguntas directivas que iniciaban con un por qué sobre un tema específico para favorecer el inicio de la conversación. 
Tabla 1

Categorias de estrategias argumentativas en el nivel preescolar

\begin{tabular}{|c|c|c|}
\hline $\begin{array}{c}\text { Estrategias } \\
\text { Argumentativas }\end{array}$ & Definición & Ejemplo \\
\hline $\begin{array}{l}\text { Referente de } \\
\text { autoridad }\end{array}$ & $\begin{array}{l}\text { Apelar a otra persona a la que se le } \\
\text { atribuye una característica de autoridad, } \\
\text { para justificar el punto de vista. }\end{array}$ & $\begin{array}{l}\text { "Mi perrito corre bien rápido, mi } \\
\text { perrito y entonces dice mi mamá } \\
\text { y yo ¡Ya le dio la chiripiorca!". }\end{array}$ \\
\hline Descripción & $\begin{array}{l}\text { Expresar características y atributos } \\
\text { esenciales de personas, eventos u } \\
\text { objetos para justificar el punto de vista. }\end{array}$ & $\begin{array}{l}\text { "Mirian no quiere que raye su } \\
\text { libro, porque ella es enojona y no } \\
\text { le gusta compartir". }\end{array}$ \\
\hline Comparación & $\begin{array}{l}\text { Establecer relaciones de semejanza o } \\
\text { diferencia entre dos o más personas, } \\
\text { eventos u objetos para justificar el } \\
\text { punto de vista. }\end{array}$ & $\begin{array}{l}\text { "Los niños somos pequeños; } \\
\text { porque mis papás son grandes". }\end{array}$ \\
\hline Analogía & $\begin{array}{l}\text { Relación entre dos o más personas, } \\
\text { eventos u objetos, señalando una } \\
\text { característica esencial entre ambos, } \\
\text { para justificar el punto de vista. }\end{array}$ & $\begin{array}{l}\text { "Los paraguas sirven para } \\
\text { proteger, son buenos como los } \\
\text { superhéroes". }\end{array}$ \\
\hline Generalización & $\begin{array}{l}\text { Afirmación que se refiere a un atributo } \\
\text { que incluye a todas las personas, eventos } \\
\text { u objetos que hacen parte de un grupo, } \\
\text { para justificar el punto de vista. }\end{array}$ & $\begin{array}{l}\text { "Nosotros podemos leer; pero } \\
\text { los niños pequeños no porque } \\
\text { rompen los libros, usan babero y } \\
\text { rompen las crayolas". }\end{array}$ \\
\hline Narración & $\begin{array}{l}\text { Relatar un acontecimiento ocurrido } \\
\text { en el pasado, para justificar el punto } \\
\text { de vista. }\end{array}$ & $\begin{array}{l}\text { "Cuando llovió la gente usó } \\
\text { paraguas y se metió a sus casas". }\end{array}$ \\
\hline Anticipación & $\begin{array}{l}\text { Proyectar un hecho antes de que este } \\
\text { ocurra, tomando como referente } \\
\text { datos previos. }\end{array}$ & $\begin{array}{l}\text { "Vamos a checar su corazón } \\
\text { y su cerebro. ¡Está enferma! } \\
\text { Entonces no podrás venir a la } \\
\text { escuela porque contagiarás a los } \\
\text { niños". }\end{array}$ \\
\hline Causalidad & $\begin{array}{l}\text { Conexión entre dos o más hechos o } \\
\text { ideas para justificar un punto de vista } \\
\text { y llegar a una conclusión. }\end{array}$ & $\begin{array}{l}\text { "Yo quiero una fiesta; pero yo no } \\
\text { me quiero casar. Cuando uno se } \\
\text { casa hacen fiesta; pero le sale una } \\
\text { panza y nace un bebé". }\end{array}$ \\
\hline $\begin{array}{l}\text { Propuesta } \\
\text { alternativa }\end{array}$ & $\begin{array}{l}\text { Generación de una justificación } \\
\text { que busca solucionar un problema } \\
\text { o situación, la cual surge como } \\
\text { respuesta a una justificación dada } \\
\text { previamente por otra persona. }\end{array}$ & $\begin{array}{l}\text { "Yo le expliqué a Emilio que no } \\
\text { se enojara con Eduardo, porque } \\
\text { él no ponchó su globo; ya que el } \\
\text { globo se rompió solo porque es } \\
\text { frágil". }\end{array}$ \\
\hline
\end{tabular}




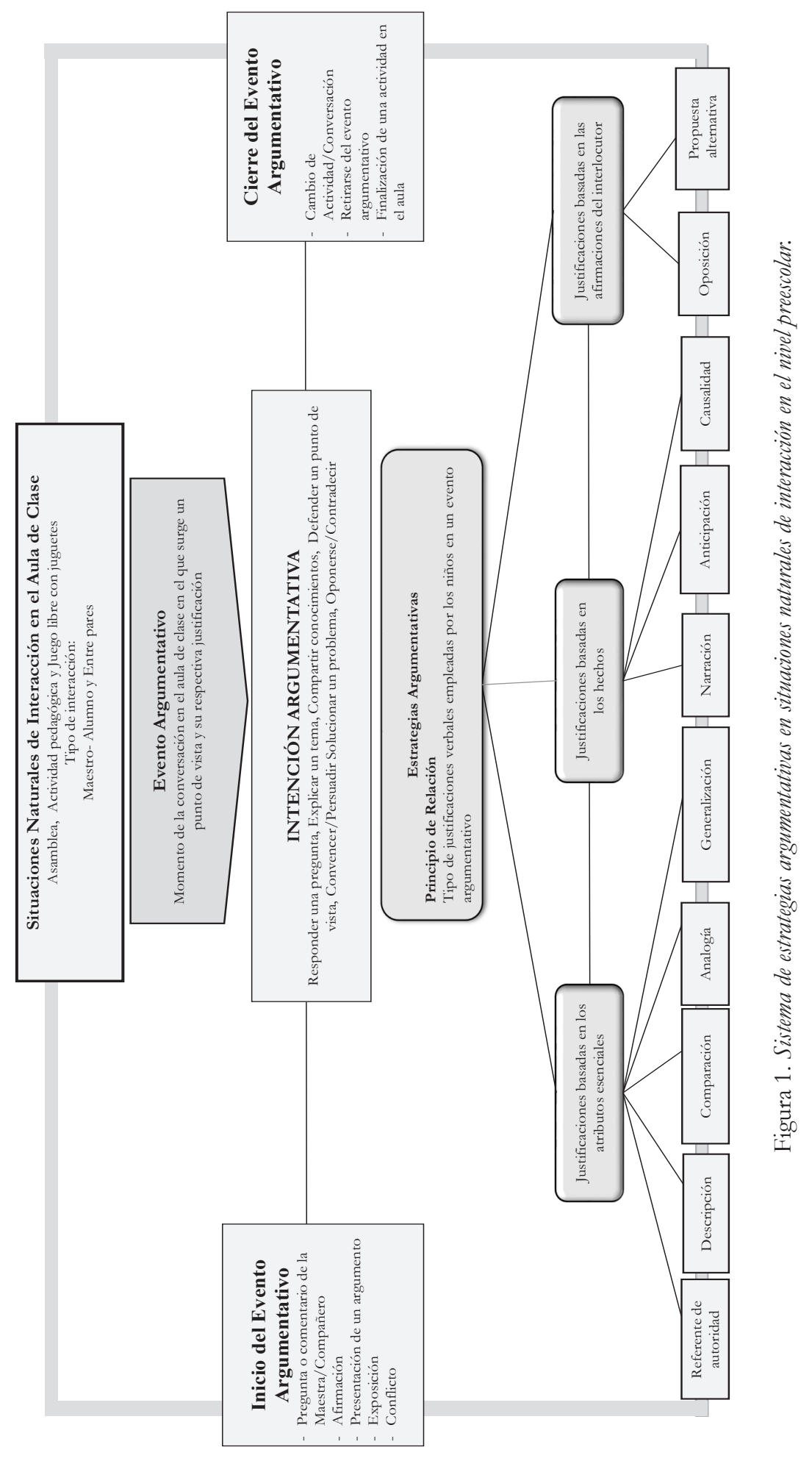

Actualidades en Psicología, 32(125), 2018, 113-131 
En la interacción entre pares fue más común que el inicio del evento se vinculara también con el comienzo de un juego simbólico. Otras razones para iniciar un evento fueron las afirmaciones de los compañeros, la lectura de cuentos y la conversación sobre temas de interés, por ejemplo, lo que hicieron el fin de semana, sus animales favoritos o un viaje familiar.

\section{Intención argumentativa}

Este elemento del sistema se vincula con el propósito que persigue el niño cuando presenta una estrategia argumentativa. En los eventos argumentativos observados se identificaron las siguientes intenciones: responder una pregunta, explicar un tema, compartir conocimientos, defender un punto de vista, convencer/persuadir, solucionar un problema, y oponerse/contradecir.

\section{Estrategias argumentativas}

El siguiente elemento lo constituyen las estrategias argumentativas que fueron clasificadas mediante el criterio del tipo de justificación que emplearon los niños para sustentar sus puntos de vista. Se trata de tres clases de justificaciones: las que están basadas en atributo esenciales, las que se relacionan con los hechos y las que retoman las afirmaciones del interlocutor.

Justificaciones basadas en atributos esenciales. Comprenden las estrategias argumentativas de: referente de autoridad, descripción, comparación, analogía y generalización. Dichas estrategias tienen en común que el niño verbaliza características que consideró relevantes de las personas, eventos u objetos para justificar su punto de vista. En los eventos documentados en este estudio, los niños enumeraban diferentes características esenciales a partir del establecimiento de semejanzas, diferencias, comparaciones, relaciones y clasificaciones.

Justificaciones basadas en los hechos. Es un grupo compuesto por las estrategias de: narración, anticipación y causalidad, las cuales presentan un tipo de justificación en donde la evidencia principal es un hecho, así como sus relaciones. En este tipo de estrategias, los niños retomaban las experiencias que habían vivido con sus familias o en la escuela para justificar, convencer sobre un tema o explicarlo. Del mismo modo, la construcción de la estrategia implicaba procesos de abstracción de la información y el establecimiento de relaciones entre los hechos.

Justificaciones basadas en las afirmaciones del interlocutor. Este tercer grupo está integrado por las estrategias de: oposición y propuesta alternativa, las cuales tienen en común que la justificación se estructura a partir de los datos previos que brinda el interlocutor. Recurrir a esta justificación implicó que los niños escucharan el argumento del otro (maestra o compañero) para estructurar, a partir de este, el argumento propio, el cual podía tener diferentes intenciones como presentar un desacuerdo o generar algún tipo de negociación para solucionar un problema.

\section{Cierre del evento argumentativo}

Es el último elemento del sistema y permite identificar cómo concluye la conversación y el efecto obtenido con la estrategia argumentativa. En esta investigación fue muy usual que 
el cierre se diera por elementos circunstanciales como la interrupción de la actividad por la llegada de una persona al aula de clase, la activación de señales de emergencia (por ejemplo, alertas sísmicas) o la terminación del tiempo de una actividad. Asimismo, en la interacción entre pares, era muy común el cambio de conversación entre los niños, el ignorar lo dicho por el compañero o retirarse del lugar donde estaba ocurriendo la conversación.

Con la finalidad de mostrar cómo se interrelacionan los elementos del Sistema de estrategias argumentativas, se presentan a continuación dos ejemplos de situaciones naturales dentro del aula de clase en las que emergieron estrategias argumentativas: conversación sobre el "Día de Muertos" y conversación sobre una fiesta.

Estrategias argumentativas durante la interacción maestro-alumno: Conversación sobre el "Día de Muertos"

Este evento ocurrió durante la actividad de asamblea de un grupo de niños del grado tercero de preescolar. Los participantes estaban sentados en círculo con su maestra y comenzaron a compartir sus conocimientos sobre la celebración del "Día de Muertos" una fiesta muy importante en la cultura mexicana. En ese momento, se dio una diferencia de puntos de vista entre dos niños en relación con el "Día de Muertos" y el "Halloween" (véase tabla 2).

En este evento surgieron cuatro tipos de estrategias argumentativas: oposición, descripción, causalidad y narración. En el turno conversacional 3, Sebastián utiliza la estrategia de oposición para expresar su desacuerdo con la afirmación que realiza Karla, ya que para él existe una diferencia entre "Día de Muertos" y "Halloween". Esta diferencia la explica empleando la estrategia de descripción, en la que expresó las siguientes características representativas del "Día de Muertos": ofrendas, veladoras, comida y frutas.

En el turno conversacional 5, Sebastián vuelve a utilizar la estrategia argumentativa de oposición, para seguir aclarando a Karla las diferencias entre estas dos celebraciones. El evento continúa a partir de la pregunta de la maestra sobre “¿Qué es una ofrenda?”. La respuesta a esta pregunta la realiza Sebastián en el turno conversacional 7, empleando la estrategia de causalidad en la que presenta una serie de hechos que están relacionados entre sí y explican la razón por la que una ofrenda en México es "para que se la coman los muertos".

Posteriormente, ante la pregunta de la maestra “¿Para quiénes será la ofrenda de sus casas?", Sebastián responde empleando la estrategia de narración en el turno conversacional 9, remitiéndose al hecho de que es para su 'Tía Conchi', que murió de una enfermedad. Finalmente, en el turno conversacional 11, el niño expresa una estrategia de oposición, para contradecir la afirmación de Karla, indicando que la ofrenda de su casa no será para sus abuelos porque su evidencia es que "no están muertos"; entonces explica que será para su bisabuelo 'Papá Pablo', que ya murió.

\section{Estrategias argumentativas durante la interacción entre pares: Conversación sobre una fiesta}

En un momento de juego libre con juguetes, se encuentran dos niñas de segundo de preescolar jugando con un muñeco al que le dicen bebé y una de ellas asume el rol de ser su mamá. En ese momento, llega su compañera Sofía que jugaba en otro rincón del espacio de juego libre para invitar a las dos niñas a su fiesta, esta invitación generó el evento argumentativo descrito en la tabla 3. 


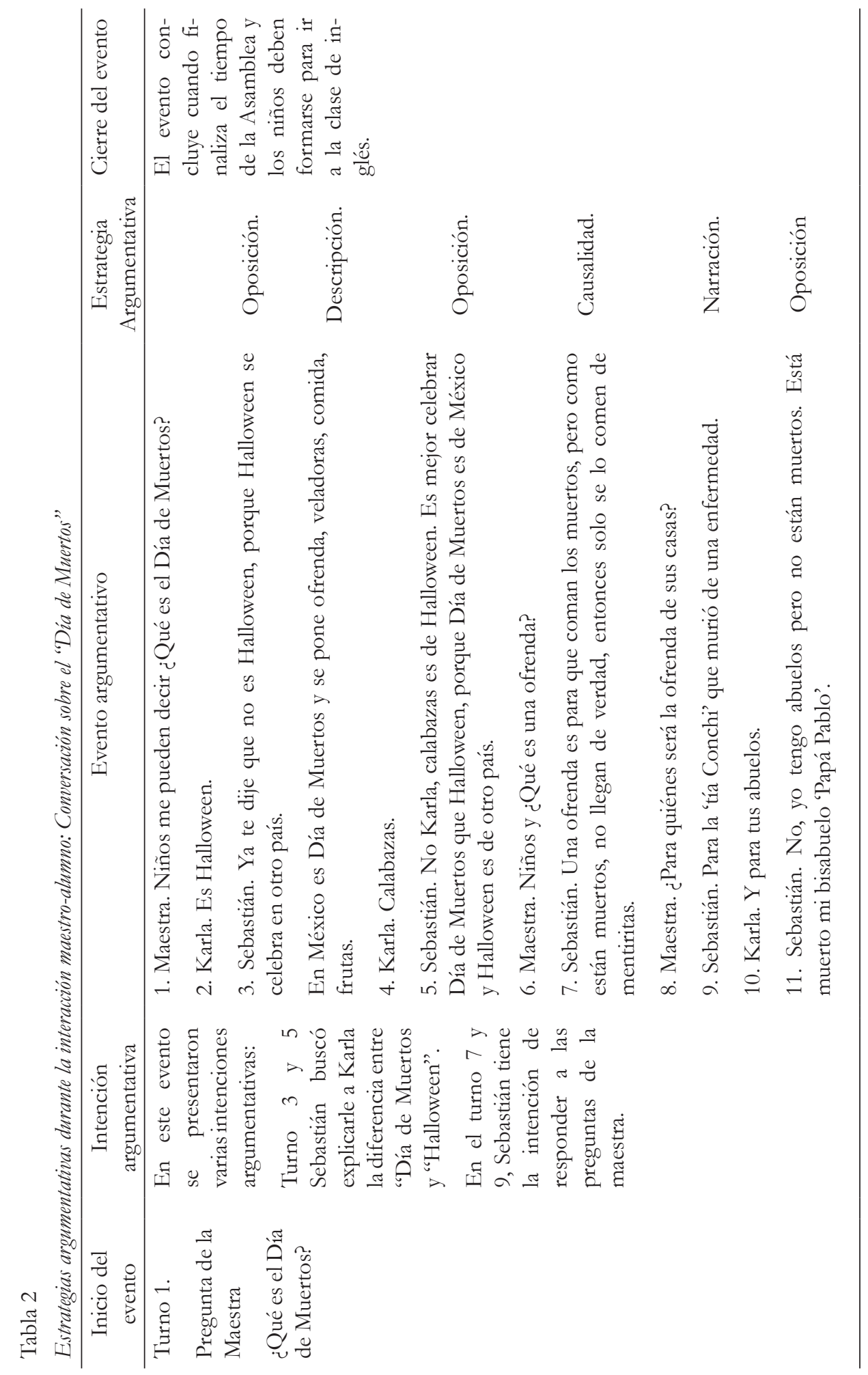




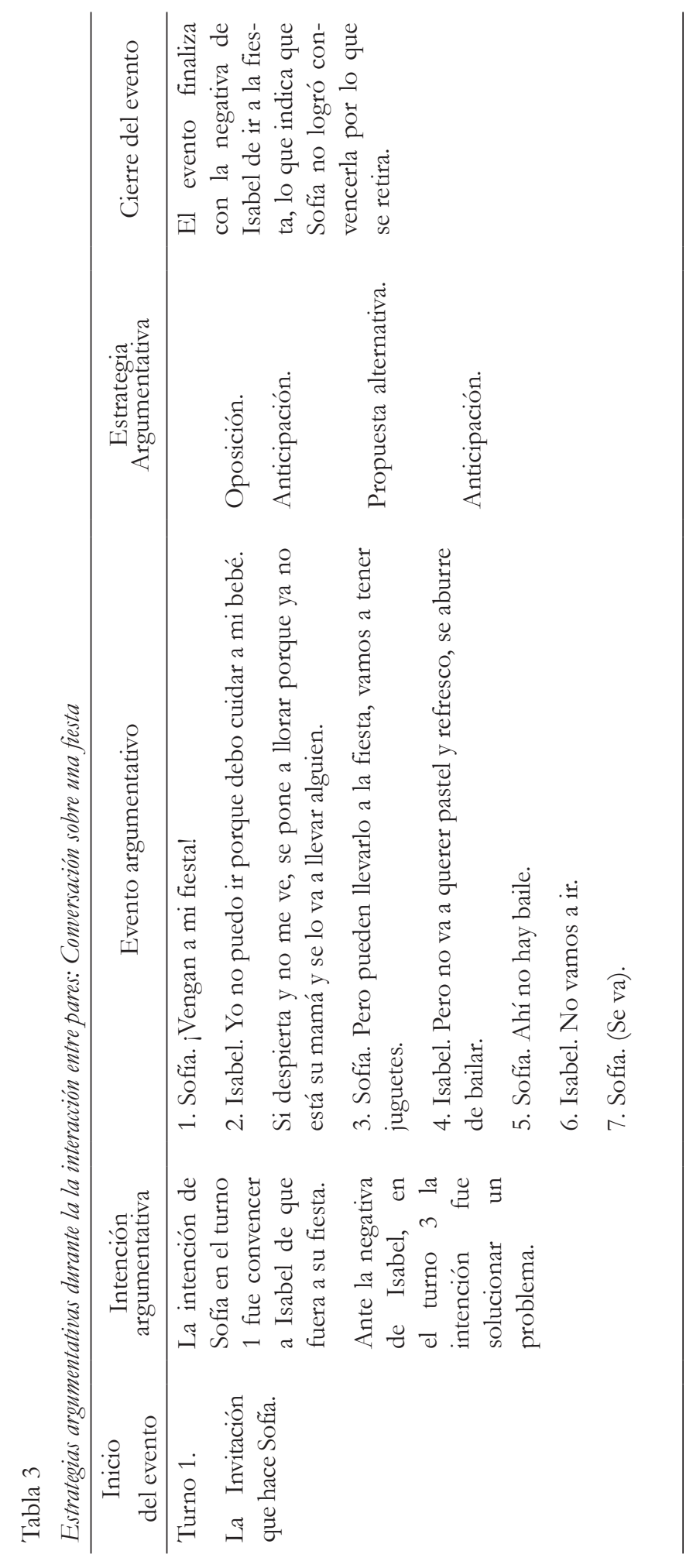

Actualidades en Psicología, 32(125), 2018, 113-131 
En este evento, se presentaron tres tipos de estrategias argumentativas: oposición, anticipación y propuesta alternativa. La estrategia de oposición apareció en el turno conversacional 2, ante la negativa de Isabel de ir a la fiesta, quien además anticipa lo que sucedería sí el bebé se despierta y ella no está. Frente a lo expuesto por su compañera, Sofía presentó en el turno 3 la estrategia de propuesta alternativa, señalando una posible solución al problema: "Pero pueden llevarlo a la fiesta, vamos a tener juguetes".

No obstante, la solución propuesta no logra convencer a Isabel quien anticipa nuevamente que su bebé no va a querer estar en la fiesta: "Pero no va a querer pastel y refresco, se aburre de bailar". Frente a esto, Sofía responde con una afirmación: "Ahí no hay baile", mostrando de nueva cuenta un interés porque Isabel vaya a su fiesta, pero ante la negativa de ella, decide retirarse.

\section{Discusión}

Los resultados obtenidos en este estudio evidencian que los participantes con edades entre los 3 y 5 años generaron diversas estrategias argumentativas en las situaciones naturales de interacción en el aula que fueron observadas: asamblea, actividad pedagógica y juego libre con juguetes. Estos hallazgos coinciden con los estudios empíricos sobre argumentación en primera infancia, los cuales identificaron, desde la edad de los tres años, habilidades en los niños para justificar sus puntos de vista en conversaciones con sus maestros y entre pares (Banks-Leite, 1997; Eisenberg, 1987; González, 2014; Kuo, 2011; Macedo, 2011; Stein \& Albro, 2001; Köymen et al., 2014).

Kuhn y Udell (2003) y Kuhn et al. (2016) afirmaron que, cuando las personas participan en diálogos argumentativos, utilizan como medios de justificación de sus argumentos, estrategias que les sirven para fortalecer sus puntos de vista. En este estudio se identificó que el empleo de estrategias argumentativas implicó que los niños participantes fueran capaces de formular un argumento cuya justificación lograra ir más allá de respuestas como: "No", "Porque sî", "Porque me gusta", siendo capaces de razonar una respuesta.

En este sentido, en los resultados se identificó que cuando los niños desplegaban una estrategia argumentativa, ponían en marcha procesos de razonamiento que se veían reflejados en sus justificaciones al momento de describir, comparar, relacionar, abstraer información y relacionar hechos. De igual manera, construir una estrategia implicaba en los niños un esfuerzo cognoscitivo para elaborar una respuesta tomando en cuenta a su interlocutor, el tema de la conversación y sus conocimientos previos al respecto. Este hallazgo coincide con los trabajos de Muller et al. (2009), quienes identificaron que la participación de los niños en situaciones de interacción argumentativa implica la habilidad de considerar el punto de vista de otra persona en lugar de solo tomar en cuenta el propio punto de vista como único referente válido, así como proporcionar justificaciones y pruebas que sustenten sus afirmaciones.

Un análisis fino de los resultados permitió la construcción de un Sistema de estrategias argumentativas, el cual es un aporte de este trabajo al estudio de la argumentación en la primera infancia. Lo anterior porque se logró identificar que las estrategias se pueden comprender de una manera más integral y contextualizada cuando se analizan en 
función de la situación de interacción en las que tienen lugar. Aunado a ello, fue posible señalar elementos importantes como el turno conversacional que dio inicio al evento argumentativo, la intención argumentativa que tuvieron los niños al desplegar determinada estrategia y la manera en la que concluye el evento.

En relación con las situaciones de interacción maestro-alumno, se identificó que cuando las maestras se mostraban interesadas por escuchar a los niños y les pedían explicaciones, generaban un ambiente propicio para que ellos argumentaran y desplegaran algún tipo de estrategia, lo cual coincide con los estudios de González (2007; 2014) y Dovingo (2016), quienes encontraron una relación entre los estilos de diálogo de las maestras con la construcción de estrategias argumentativas por parte de sus alumnos.

En torno a la interacción entre pares, este estudio encontró que la actividad de juego libre con juguetes fue el momento más propicio para observar la construcción de estrategias argumentativas entre los niños. Se halló que los momentos lúdicos dentro del aula de clase, que brindan a los niños la libertad de elegir materiales y juguetes de su interés, posibilitaron la creación de juegos simbólicos con sus compañeros; en estos juegos, los niños representaban personajes que tenían diálogos entre sí y, dentro del curso de la actuación, se veían en la necesidad de justificar sus puntos de vista y comportamientos. Al respecto, Stein y Migdalek (2017) afirmaron que, en el juego simbólico, los niños emplean diversos recursos del lenguaje para construir la dramatización de acciones y crear diálogos ficcionales dándoles voz a los personajes, quienes expresan en el juego sus puntos de vista.

En relación con el componente de estrategias argumentativas, el sistema las agrupó según los tipos de justificación empleados por los participantes. Cabe mencionar que, en la literatura revisada en este estudio, no existe consenso sobre el criterio para clasificar las estrategias argumentativas. Migdalek et al. (2014a) emplearon el criterio de la complejidad argumentativa, tomando como referencia la nueva información que emplean los niños para justificar un punto de vista.

Ahora bien, a pesar de que los hallazgos del estudio de Migdalek et al. (2014a) mostraron que, desde el punto de vista cognoscitivo, una estrategia como la propuesta alternativa implica un razonamiento más complejo que la estrategia de referente de autoridad, se tomó la decisión de iniciar con la comprensión de las estrategias en relación con los tipos de justificación empleados por los niños en las situaciones naturales de interacción en el aula, para posteriormente profundizar en futuros estudios en esa complejidad. Se prefirió hacerlo de este modo ante la escasa evidencia empírica sobre la argumentación en niños mexicanos.

Respecto a las estrategias documentadas, el primer grupo correspondió a aquellas basadas en atributos esenciales, que tienen en común el empleo de descripciones, establecimiento de semejanzas y diferencias, comparaciones entre objetos, personas o eventos, así como la construcción de relaciones y clasificaciones. Al respecto, Vigotsky (1934/2009) afirmó que, cuando un niño emplea el lenguaje oral con una intención comunicativa, por ejemplo, resolver un problema, emprende una serie de operaciones mentales que les sirven a sus propósitos, como el agrupamiento en familias de conceptos mutuamente relacionados, también la creación de categorías y generalizaciones, teniendo en cuenta un rasgo común. 
Asimismo, en el segundo grupo de estrategias, los niños fueron capaces de realizar narraciones, anticipaciones y establecer relaciones de causalidad, las cuales eran empleadas como una evidencia de la veracidad de sus afirmaciones. En este sentido, los participantes retomaron los conocimientos previos y las experiencias que han vivido con sus familias o en la escuela, para justificar sus puntos de vista. Autores como Dovingo (2016), Macedo (2011) y Zadunaisky y Blum-Kulka (2010) denominaron este conocimiento como un "terreno social común" que les permite a los niños razonar conjuntamente y construir argumentos en la conversación.

Una contribución de este estudio al estado del arte de las estrategias argumentativas en el nivel preescolar es la estrategia de causalidad, la cual es una evidencia de la manera en que la argumentación está vinculada con la actividad cognoscitiva en la infancia. Esto es así porque implica un refinamiento de la justificación proporcionada por los niños, mostrando su capacidad para comunicar las causas y relaciones lógicas que permiten explicar un fenómeno o situación, y como resultado llegar a una conclusión.

El grupo de estrategias en donde el tipo de justificación se basó en las afirmaciones del interlocutor, es un indicador importante de cómo los niños, desde temprana edad, son capaces de tomar en cuenta las afirmaciones y argumentos del otro para construir sus propios razonamientos, expresar una oposición o buscar llegar a consensos entre puntos de vista diferentes (Dovingo, 2016; Muller et al., 2009).

Cuando se analizó la intención argumentativa de los niños que participaron en este estudio, se identificó que ellos no solo emplearon las estrategias argumentativas con la intención de convencer o persuadir a otros en situaciones de disputa, como se ha documentado en distintas investigaciones sobre argumentación infantil (Stein \& Albro, 2001; Kuo, 2011; Macedo, 2011; Migdalek et al., 2014a). También, los participantes argumentaban para construir un conocimiento compartido sobre un tema específico, responder una pregunta, complementar las ideas de sus compañeros y solucionar un problema, lo cual refleja el vínculo de la argumentación con el razonamiento conjunto que emerge en la interacción social y brinda mayores explicaciones al interrogante de por qué argumentan los niños.

\section{Limitaciones y contribuciones}

Este estudio presenta limitaciones relacionadas con el alcance del tipo de investigación. $\mathrm{Al}$ ser un análisis cualitativo, sus resultados no pueden ser generalizables y una réplica del estudio precisa la necesidad de contar con interobservadores, para así fortalecer los niveles de confiabilidad y validez del Sistema de las estrategias argumentativas.

Otra limitación fue que no se llevaron a cabo entrevistas a profundidad con las maestras participantes con el fin de que permitieran comprender sus concepciones sobre la argumentación en el nivel preescolar y la relación de dicha concepción con su práctica educativa. Se considera que en futuras investigaciones se emplee este tipo de técnica para complementar el análisis de la interacción maestro-alumno.

Más allá de las limitaciones antes mencionadas, este estudio proporciona nueva evidencia empírica que reafirma la emergencia de la actividad argumentativa en el nivel preescolar. Además, profundizando en la relación de diversos elementos involucrados con la 
construcción de las estrategias argumentativas, los cuales quedaron representados en un sistema que brinda una comprensión más compleja y dinámica de las diversas situaciones de interacción en las que dichas estrategias se construyen.

Las implicaciones prácticas de este estudio se vinculan con la documentación de diversas situaciones del aula de clase como: la asamblea, la actividad pedagógica y el juego libre con juguetes. Estas actividades se relacionaron con la emergencia de estrategias argumentativas; además, al analizar las interacciones maestro-alumno y entre pares, se aportan datos específicos sobre las características de dichas interacciones y las intervenciones de los docentes que promueven el diálogo argumentativo.

En síntesis, se concluye que el estudio de la argumentación en niños preescolares mexicanos en un terreno de investigación fértil e incipiente que requiere ser fortalecido con futuros estudios de tipo cualitativo, cuantitativo y mixtos que ahonden en la comprensión de este constructo en la primera infancia. En investigaciones futuras, se considera analizar el criterio de complejidad de las estrategias argumentativas, así como la relación entre las estrategias y el grado escolar, con el objetivo de comprender si existen diferencias en el empleo de las estrategias en función de las experiencias de interacción y las actividades curriculares que tienen los niños en cada grado del nivel preescolar.

\section{Referencias}

Álvarez-Gayou, J. L. (2011). Cómo hacer investigación cualitativa. México, D. F., México: Paidós.

Banks-Leite, L. (1997). La argumentación en el niño de edad preescolar. Revista Colombiana de Psicología, 5(6), 91-95. doi: 10.15446/rcp

Cisterna, V., \& Garayzábal, E. (2016). La argumentación infantil: perspectivas analíticas y evaluativas. Una mirada crítica desde un enfoque integral. Revista Akademéia, 7(1), 3152. Recuperado de: http:/ / revistas.ugm.cl/index.php/rakad/article/view/136

Coffey, A., \& Atkinson, P. (2004). Encontrar el sentido a los datos cualitativos. Medellín, Colombia: Universidad de Antioquia.

Coirier, P., \& Golder, C. (1993). Writing Argumentative Text: A Developmental Study of the Acquisition of Supporting Structures. European Journal of Psychology of Education, 7(2), 169-181. doi: 10.1007/BF03173160

Dolz, J., \& Pasquier, A. (2000). Escribo mi opinión: una secuencia didáctica de iniciación a los textos de opinión para el tercer ciclo de educación primaria. Navarra, Gobierno de Navarra: Departamento de Educación y Cultura.

Dovingo, F. (2016). Argumentation in preschool: a common ground for collaborative learning in early childhood. European Early Childhood Education Research Journal, 24(6), 818-840, doi: 10.1080/1350293X.2016.1239327

Eisenberg, A. R. (1987). Learning to Argue with Parents and Peers. Argumentation, 1(2), 113-125. doi: 10.1007/BF00182256

Flick, U. (2007). Introducción a la investigación cualitativa. Madrid, España: Ediciones Morata. 
González, J. (2007). La argumentación a partir de cuentos infantiles. Revista Mexicana de Psicología, 12(33), 657-677. Recuperado de http://www.comie.org.mx/ documentos/rmie/v12/n033/pdf/N33J.pdf

González, J. (2014). El proceso de argumentación en el inicio escritor. Acta Universitaria, 24(5), 33-46. doi: 10.15174.au.2014.633

Kerlinger, F. N., \& Lee, H. B. (2002). Investigación del comportamiento. México D. F., México: McGraw-Hill.

Köymen, B., Rosenbaum, L., \& Tomasello, M. (2014). Reasoning during joint decisionmaking by preschool peers. Cognitive Development, 32, 74-85. doi: 10.1016/j. cogdev.2014.09.001

Kuo, M. (2011). Children in arguments with peers: Young children's strategies as Opposer and Opposee. Proceedings of the 23rd North American Conference on Chinese Linguistics, 23(2), 123-132. Recuperado de https:// naccl.osu.edu/sites/naccl.osu.edu/files/ NACCL-23_2_09.pdf

Kuhn, D. (1993). Science as argument: implications for teaching and learning scientific thinking. Science Education Hoboken, 77(3), 319-337.

Kuhn, D., \& Udell, W. (2003). The Development of Argument Skill. Child Development, 74(5), 1245-1260. Recuperado de: http://www.jstor.org/stable/3696176

Kuhn, D., Hemberger, L., \& Khait, V. (2016). Dialogic argumentation as a bridge to argumentative thinking and writing. Infanciay Aprendizaje, 39(1), 25-48. doi: 10.1080/02103702.2015.1111608

Macedo, A. P. (2011). The Development of Children's Argument Skills (Thesis doctoral). University of London, London. Recuperado de https://core.ac.uk/download/pdf/28897840.pdf

Márquez, A. (2016). Promoción de estrategias argumentativas en la solución de conflictos de manera independiente en niños preescolares (Tesis de Maestría inédita). Universidad Nacional Autónoma de México, México.

Martí, E. (1978). Estudio del Pensamiento analógico en el niño de 2 a 8 años: Metáforas y comparaciones espontáneas. Amuario de psicología, 19, 39-56. Recuperado de: raco.cat/index. php/AnuarioPsicologia/article/view/64452/88133

Mercer, N. \& Littleton, K. (2007). Dialogue and the Development of Children's Thinking. London: Routledge.

Mercer, N. (2011). Reasoning serves argumentation in childrens. Cognitive Development, 26(3), 177- 191. doi: 10.1016/j.cogdev.2010.12.001

Migdalek, M. J., \& Rosemberg, C. R. (2013). Construcción multimodal de los argumentos de niños pequeños en disputas durante situaciones de juego. Papeles de trabajo sobre Cultura, Educación y Desarrollo humano, 9(4), 1-16. Recuperado de: https:/ /www.uam.es/otros/ptcedh/2013v9_ pdf/v9n4esp.pdf

Migdalek, M. J., Santíbañez, S., \& Rosemberg, C. R. (2014a). Estrategias argumentativas en niños pequeños: Un estudio a partir de las disputas durante el juego en contextos escolares. Revista Signos, 47(86), 435-462. doi: 10.4067/S0718-09342014000300005 
Migdalek, M. J., Santibañez, S., \& Rosemberg, C. R. (2014b). La Génesis de la Argumentación. Un Estudio con Niños de 3 a 5 Años en Distintos Contextos de Juego. Íkala, Revista de Lenguaje y Cultura, 19(3), 251-267. doi: 10.17533/udea.ikala.v19n3a03

Muller, N., Perret-Clermont, A. N., Tartas, V., \& Iannaccone, A. (2009). Psychosocial Processes in Argumentation. En: N. Muller \& A. Perret-Clermont (Eds.), Argumentation and education (pp. 67-90). New York: Springer.

National Association for the Education of Young Children. (2011). Código de conducta ética y declaración de compromiso. Estados Unidos: Autor. Recuperado de http://www. psicologia.unam.mx/documentos/pdf/comite_etica/NAEYC_Ethics_Spanish_ Position_Statement2011_09202013.pdf

Rapanta, C., García-Mila, M., \& Gilabert, S. (2013). What is Meant by Argumentative Competence? An Integrative review of Methods of Analysis and Assessment in Education. Review of Educational Research, 83(4), 483-520.

Rodríguez, S., Lorenzo, O., \& Herrera. L. (2005). Teoría y práctica del análisis de datos cualitativos. Proceso general y criterios de calidad. Revista Internacional de Ciencias Sociales y Humanidades, 15(2), 133-154. Recuperado de http://www.redalyc.org/ pdf/654/65415209.pdf

Ruiz, J. L. (1996). Metodología de la investigación cualitativa. Bilbao: Universidad de Deusto.

Silvestri, A. (2001). La producción de la argumentación razonada en el adolescente: Las falacias de aprendizaje. En M. C. Martínez (Ed.) Aprendizaje de la argumentación razonada. Desarrollo temático de los textos expositivos y argumentativos (pp. 29-48). Cali: Cátedra UNESCO para la Lectura y la Escritura, Universidad del Valle.

Sociedad Mexicana de Psicología. (2009). Código ético del psicólogo. México D. F., México: Trillas.

Stein, N., \& Albro, E. R. (2001). The origins and nature of arguments: Studies in conflict understanding, emotion, and negotiation. Discourse Processes, 32(2-3), 113-33. doi: 10.1080/0163853X.2001.9651594

Stein, A., \& Migdalek, M. J. (2017). La construcción del "mundo de ficción” y de la trama narrativa en situaciones de juego simbólico en el hogar. Actualidades en Psicología, 31(122), 1-15. doi: 10.15517/ap.v31i122.22707

Vigotsky, L. S. (2009). Pensamiento y lenguaje. [Trabajo original publicado 1934]. México D. F., México: Ediciones Quinto Sol.

Zadunaisky, E. S., \& Blum-Kulka, S. (2010). Peer talk as a 'double opportunity space': The case of argumentative discourse. Discourse in Society, 21(2), 211-233. doi: $10.1177 / 095792650935384$

Recibido: 15 febrero de 2018

Aceptado: 16 de septiembre de 2018 